\title{
Endothelial Interferon Regulatory Factor 1 Regulates Lipopolysaccharide-Induced VCAM-1 Expression Independent of NFKB
}

\author{
Rui Yan ${ }^{a}$ Matijs van Meurs ${ }^{a}$ b Eliane R. Popa ${ }^{a}$ Rianne M. Jongman ${ }^{a-c}$ \\ Peter J. Zwiers ${ }^{a}$ Anita E. Niemarkt ${ }^{a}$ Timara Kuiper $^{\mathrm{a}}$ Jan A. Kamps ${ }^{\mathrm{a}}$ \\ Peter Heeringa $^{a}$ Jan G. Zijlstrab Grietje Molema ${ }^{a}$ Jill Mosera, b \\ a Medical Biology Section, Department of Pathology and Medical Biology, ${ }^{b}$ Department of Critical Care, and \\ ${ }^{c}$ Department of Anaesthesiology, University of Groningen, University Medical Center Groningen, \\ Groningen, The Netherlands
}

\section{Keywords}

Interferon regulatory factor $1 \cdot$ Sepsis - Lipopolysaccharide Endothelial cells $\cdot$ Inflammation $\cdot$ NFKB signaling

\begin{abstract}
Sepsis is a severe systemic inflammatory response to infection. Endothelial activation and dysfunction play a critical role in the pathophysiology of sepsis and represent an important therapeutic target to reduce sepsis mortality. Interferon regulatory factor 1 (IRF-1) was recently identified as a downstream target of TNF-a-mediated signal transduction in endothelial cells. The aim of this study was to explore the importance of IRF-1 as a regulator of lipopolysaccharide (LPS)-induced endothelial proinflammatory activation. We found that renal IRF-1 was upregulated by LPS in vivo as well as in LPS-stimulated endothelial cells in vitro. Furthermore, we identified intracellular retinoic acid inducible gene-I (RIG-I) as a regulator of LPS-mediated IRF-1 induction. IRF-1 depletion specifically resulted in diminished induction of VCAM-1 in response to LPS, but not of E-selectin or ICAM-1, which was independent of NFKB signaling. When both IRF-1 and the RIG-I adapter protein mitochondrial antiviral signal-
\end{abstract}

\section{KARGER}

E-Mail karger@karger.com

www.karger.com/jin ing (MAVS) were absent, VCAM-1 induction was not additionally inhibited, suggesting that MAVS and IRF-1 reside in the same signaling pathway. Surprisingly, E-selectin and IL-6 induction were no longer inhibited by MAVS knockdown when IRF-1 was also absent, revealing a redundant endothelial activation pathway. In summary, we report an IRF-1-mediated proinflammatory signaling pathway that specifically regulates LPS-mediated VCAM-1 expression, independent of NFKB.

(c) 2017 S. Karger AG, Basel

\section{Introduction}

Sepsis is a severe life-threatening condition that arises when the host's systemic inflammatory response to an infection impairs its own tissues resulting in organ dysfunction [1-3]. In the United States, more than 750,000 patients are diagnosed with severe sepsis each year, and more than 250,000 sepsis deaths make sepsis the major cause of mortality in critically ill patients $[4,5]$. Sepsisinduced acute kidney injury is a common problem in critically ill patients. Although our understanding of the 
exact pathophysiology of sepsis-induced acute kidney injury is incomplete, the endothelium is increasingly recognized as an active contributor to the morbidity and mortality associated with sepsis, due to exaggerated endothelial activation and vascular leakage [6]. Activated endothelial cells promote the transmigration of leukocytes from the blood into underlying tissues through endothelial cell-leukocyte interactions, a process that can be detrimental to organ function $[7,8]$. Understanding the mechanisms underlying endothelial activation will enable us to identify new therapeutic targets to influence vascular behavior in sepsis and reduce sepsis mortality.

Lipopolysaccharide (LPS) is the main structural component of the Gram-negative bacterial cell wall and a critical component in the pathogenesis of sepsis since it leads to activation of endothelial cells $[8,9]$. The longstanding paradigm suggests that LPS initiates downstream signaling by binding to Toll-like receptor 4 (TLR4) together with the adapter protein MD2. Intracellular downstream signaling leads to activation of I $\mathrm{KB}$ kinase, which in turn causes rapid phosphorylation and degradation of $I \kappa B$ proteins resulting in the activation of NFKB [10]. NFKB controls the transcription of many proinflammatory genes, including endothelial adhesion molecules, cytoand chemokines $[11,12]$. Recent work from our laboratory identified retinoic acid inducible gene-I (RIG-I) as an additional regulator of endothelial proinflammatory activation that functions in parallel with TLR4 [13].

Interferon regulatory factor 1 (IRF-1) is a member of the interferon (IFN) regulatory transcription factor family and was originally described as a transcription factor that can activate type I IFN genes [14]. IRF-1 is expressed at low levels in quiescent cells and its expression is increased by various stimuli, such as types I and II IFN, LPS, IL-1, IL-6, TNF- $\alpha$, and viral infection $[15,16]$. IRF-1 is either a transcriptional activator or a repressor of target genes that plays key roles in anti-infection immune responses, the regulation of oncogenes, cell proliferation, proinflammatory gene expression, and the development of the immune system [16, 17]. Moreover, IRF-1 gene induction in the renal tubules by reactive oxygen species was shown to be an early event that exacerbated injury and promoted inflammation in murine ischemia/reperfusion injury [18]. Full-body IRF-1 knockout mice were protected from lethal endotoxemia by an inhibitory effect on LPS-mediated proinflammatory cytokine production [19].

In endothelial cells, IRF-1 was recently shown to regulate TNF- $\alpha$-mediated signaling, which specifically regulated the expression of the adhesion molecule VCAM-1
$[20,21]$. Recently, we also showed that RIG-I can control VCAM-1 expression in response to LPS challenge [13]. In this study we investigated whether endothelial IRF-1 plays a role in the regulation of LPS-mediated endothelial proinflammatory activation, and explored molecular mechanisms of the regulatory pathways involved.

\section{Materials and Methods}

Mice

Male C57Bl6 mice (Harlan, Horst, The Netherlands) were housed in a specific pathogen-free facility, maintained on chow and water ad libitum, and housed in temperature-controlled chambers $\left(24^{\circ} \mathrm{C}\right)$ with a 12 -h light/dark cycle. The mice were challenged via intraperitoneal (i.p.) injection of LPS $(0.5$ or $1 \mathrm{mg} / \mathrm{kg}$, Escherichia coli, serotype O26:B6 (15,000 EU/g; Sigma-Aldrich, St. Louis, MO, USA). Mice were sacrificed 4, 8, and $24 \mathrm{~h}$ after LPS administration. Control mice were administered the same volume of in $0.9 \% \mathrm{NaCl}$ i.p. Prior to sacrifice, mice were anaesthetized with $\mathrm{O}_{2}$ /isoflurane and blood was collected in EDTA tubes. Organs were harvested, snap-frozen in liquid nitrogen and stored at $-80^{\circ} \mathrm{C}$ until analysis. All procedures were performed according to Dutch laws and international guidelines on animal experimentation, and were approved by the Animal Ethics Committee of the University of Groningen.

\section{Endothelial Cell Culture and Stimulation}

Human umbilical vein endothelial cells (HUVEC) were obtained from Lonza (Breda, The Netherlands) and cultured in culture plates (Costar; Corning, New York, USA) in EBM-2 medium supplemented with EGM-2 MV SingleQuot Kit Supplements and Growth Factors (Cat. No. CC-3202, Lonza, The Netherlands). Endothelial cell cultures were used at passage 5-6 in all experiments. Immortalized HL-60 leukocytes, kindly provided by G. Fey (University of Erlangen, Germany), were maintained in RPMI 1640 medium supplemented with $10 \%$ fetal bovine serum. Primary peritubular endothelial cells from the kidney cortex were isolated from C57Bl6 mice using anti-CD31 Ab-conjugated beads, and cultured as described previously [22]. For siRNA interference, endothelial cells were plated at a density of $20,000 \mathrm{cells} / \mathrm{cm}^{2}$. For all other experiments, HUVEC were grown to confluence before the start of the experiment. Cells were stimulated for $4 \mathrm{~h}$ with LPS dissolved in $0.9 \% \mathrm{NaCl}$ to a final concentration of $1 \mu \mathrm{g} / \mathrm{ml}$, unless indicated otherwise. All cells were cultured at the UMCG Endothelial Cell Facility.

\section{siRNA-Mediated Gene Silencing}

FlexiTube small interfering RNA sequences for human IRF-1, RIG-I, and mitochondrial antiviral signaling (MAVS) were purchased from Qiagen (Leusden, The Netherlands). Three sequences per target were tested for their knockdown efficiency and the siRNA sequence creating the highest knockdown was used for all subsequent experiments. AllStars negative control siRNA (Qiagen) was used in all knockdown experiments. Transient transfections were performed using Lipofectamine 2000 Reagent (Life Technologies, Carlsbad, CA, USA), according to the manufacturer's instructions. Forty-eight hours after transfection, the cells 
were incubated with LPS for the times indicated, and subsequently analyzed by RT-qPCR and Western blot to confirm downregulation of the target genes and our genes of interest. Transfection with scrambled siRNA did not result in major activation of the endothelial cells under basal conditions (see online suppl. Fig. 1; see www.karger.com/doi/10.1159/000477211 for all online suppl. material).

\section{Laser Microdissection of the Renal Microvasculature}

Nine-micrometer cryosections from mouse kidney were cut for laser microdissection, as previously described, to collect arteriolar, glomerular, and venous vascular segments [23]. Laser microdissected samples were collected in $0.5-\mathrm{mL}$ AdhesiveCap tubes and stored at $-80^{\circ} \mathrm{C}$ until further analysis.

\section{Gene Expression Analysis by RT-qPCR}

Total RNA from kidneys and HUVEC was isolated using the RNeasy Mini Plus Kit (Qiagen) and total RNA from laser microdissected material was isolated using RNeasy ${ }^{\circledR}$ Micro Plus Kit (Qiagen), according to the manufacturer's protocols. The integrity of RNA from kidney and HUVEC was determined by gel electrophoresis. RNA purity (OD260/OD280) and concentration (OD260) were assessed by a NanoDrop ${ }^{\circledR}$ ND-1000 UV-Vis spectrophotometer (NanoDrop Technologies, Rockland, ME, USA). RNA (max of 2,500 ng), 100 units SuperScript III RNase reverse transcriptase (Invitrogen, Breda, The Netherlands), 40 units of RNase OUT inhibitor (Invitrogen), and $250 \mathrm{ng}$ of random hexamers (Promega, Leiden, The Netherlands) were used for the synthesis of cDNA in a final volume of $20 \mu \mathrm{L}$. The Assay-on-Demand primers (Applied Biosystems, Nieuwerkerk aan den IJssel, The Netherlands) for quantitative PCR were specific for housekeeping gene GAPDH (assay ID Hs99999905_m1), IRF-1 (assay ID Hs00971960_m1), Ddx58 (RIG-I) (assay ID Hs00204833_m1), MAVS (assay ID Hs00920075_m1), E-selectin (assay ID Hs00174057_m1), ICAM-1 (assay ID Hs00164932_m1), VCAM-1 (assay ID Hs00365486_m1), IL-6 (assay ID Hs00174131_m1), and IL-8 (assay ID Hs00174103_m1). qPCR was performed in a ViiA 7 real-time PCR System (Applied Biosystems, Nieuwerkerk aan den IJssel, The Netherlands). Samples were run in duplicate, and the obtained threshold cycle values $\left(\mathrm{C}_{\mathrm{T}}\right)$ were averaged. Gene expression was normalized to the expression of the reference gene GAPDH, resulting in the $\Delta \mathrm{C}_{\mathrm{T}}$ value. The mRNA level relative to GAPDH was calculated by $2^{-\Delta C_{T}}$.

Western Blot Analysis

After being cultured under the indicated conditions, cells were washed twice with ice-cold PBS and lysed in RIPA lysis buffer (100 $\mathrm{mm} \mathrm{NaCl}, 50 \mathrm{~mm}$ Tris $\mathrm{pH}$ 8.0, 0.1\% SDS [w/v], 0.5\% [v/v] Na deoxycholate, $1 \%$ [v/v] IGEPAL) containing protease inhibitor cocktail and phosphatase inhibitors (Roche, Almere, The Netherlands). The DC Protein assay (Bio-Rad Laboratories, Hercules, CA, USA) was used to determine protein concentration, with bovine serum albumin (BSA) solution as the standard. Protein samples $(20 \mu \mathrm{g}$ protein/lane) were separated by SDS-PAGE on polyacrylamide gels and then transferred to nitrocellulose membranes (Bio-Rad Laboratories, Utrecht, The Netherlands). Blots were blocked in blocking buffer consisting of 5\% (w/v) not-fat milk (Campina, Friesland, The Netherlands) in Tris-buffered saline (TBS) with $0.1 \%(\mathrm{v} / \mathrm{v})$ Tween 20 for $1 \mathrm{~h}$. The blots were subsequently incubated with primary antibodies overnight at $4{ }^{\circ} \mathrm{C}$. The primary an- tibodies used were rabbit anti-IRF-1 (\#8478, Cell Signaling Technology, Leiden, The Netherlands), rabbit anti-IкBa (06-494, Upstate Biotechnologies, CA, USA), mouse anti-RIG-I (clone Alme-1, Enzo Life Science, Raamsdonksveer, The Netherlands), rabbit anti-MAVS (A300-782A, Bethyl Laboratories Inc., Montgomery, TX, USA), rabbit anti-E-selectin (sc-14011), rabbit antiVCAM-1 (sc-8304; Santa Cruz, Heidelberg, Germany), rabbit anti-ICAM-1 (sc-8439), and mouse anti-actin (clone C4) (MAB1501; Merck Millipore, Darmstadt, Germany). After washing with TBS/0.1\% (v/v) Tween 20 for $15 \mathrm{~min}$, blots were incubated for $1 \mathrm{~h}$ at room temperature (RT) with horseradish peroxidase-conjugated goat anti-mouse or goat anti-rabbit secondary antibodies (Southern Biotech, Birmingham, AL, USA) diluted in blocking buffer. After washing, detection was performed using Luminata Forte Western horseradish peroxidase substrate (Merck-Millipore). Images were taken using a Geldoc XR system (Bio-Rad, Veenendaal, The Netherlands). Western blot bands were quantified by densitometry and the background was subtracted using Image Lab software version 5.2.1 (Bio-Rad). After the initial proteins of interest were detected, the blots were rinsed in TBS / $0.1 \%$ (v/v) Tween 20, and then incubated with PLUS Western Blot stripping buffer (\#46430, Thermo Scientific, Rockford, USA) at RT for $30 \mathrm{~min}$. The blots were subsequently washed with TBS $/ 0.1 \%$ (v/v) Tween 20 for $10 \mathrm{~min}$. Blots were then blocked and incubated with the second set of primary and secondary antibodies, as described above.

\section{IRF-1 and NFkB p65 Nuclear Translocation}

HUVEC were cultured on sterile glass coverslips (MenzelGläser, Braunschweig, Germany) and transfected with either scrambled, IRF-1, RIG-I, or MAVS siRNA. Forty-eight hours after transfection, cells were stimulated with LPS in $0.9 \% \mathrm{NaCl}(1 \mu \mathrm{g} /$ $\mathrm{ml}$ ) for either $40 \mathrm{~min}$ or $4 \mathrm{~h}$. Cells were washed in cold PBS and fixed for $20 \mathrm{~min}$ with $1 \%(\mathrm{v} / \mathrm{v})$ formaldehyde in PBS on ice, followed by $0.25 \%(\mathrm{v} / \mathrm{v})$ Triton X-100 in PBS for $5 \mathrm{~min}$ at RT. After washing, cells were blocked with $3 \%$ (w/v) BSA in PBS for 30 min at RT and then incubated with either rabbit anti-IRF-1 antibody (\#8478) or rabbit anti-p65 antibody (\#D14E12, both from Cell Signaling Technology) diluted in washing buffer (PBS containing $0.5 \%[\mathrm{w} / \mathrm{v}]$ BSA and $0.05 \%[\mathrm{v} / \mathrm{v}]$ Tween 20$)$ for $1 \mathrm{~h}$ at RT. After washing, cells were incubated with Alexa Fluor ${ }^{\circledR} 555$-conjugated donkey anti-rabbit secondary antibody (A-31572, Life Technologies, The Netherlands) for $45 \mathrm{~min}$ at RT. The cells were again washed and mounted in Aqua/Polymount medium containing DAPI $(1.5 \mu \mathrm{g} / \mathrm{mL})$ (Polysciences, Warrington, PA, USA). Fluorescence images were taken with a Leica DM/RXA fluorescence microscope equipped with a Leica DFC450C digital camera (Wetzlar, Germany) and Leica LAS V4.2 Image Overlay Software or using a Leica DM4000B fluorescence microscope equipped with a Leica DFC345FX digital camera (Leica Microsystems Ltd., Germany) and Leica LAS V4.5 Image Software. All images were taken with equal exposure times.

\section{Localization of IRF-1 in vivo}

Five-micrometer cryosections from snap-frozen mouse kidneys were fixed in acetone for $10 \mathrm{~min}$. To detect IRF-1 and CD31, sections were incubated for $1 \mathrm{~h}$ with primary rabbit anti-IRF-1 antibody (sc-640, Santa Cruz) or rabbit anti-IRF-1 antibody (\#8478, Cell Signaling Technology), and rat anti-CD31 antibody (\#550274, BD Pharmingen, San Diego, CA, USA) in PBS contain- 


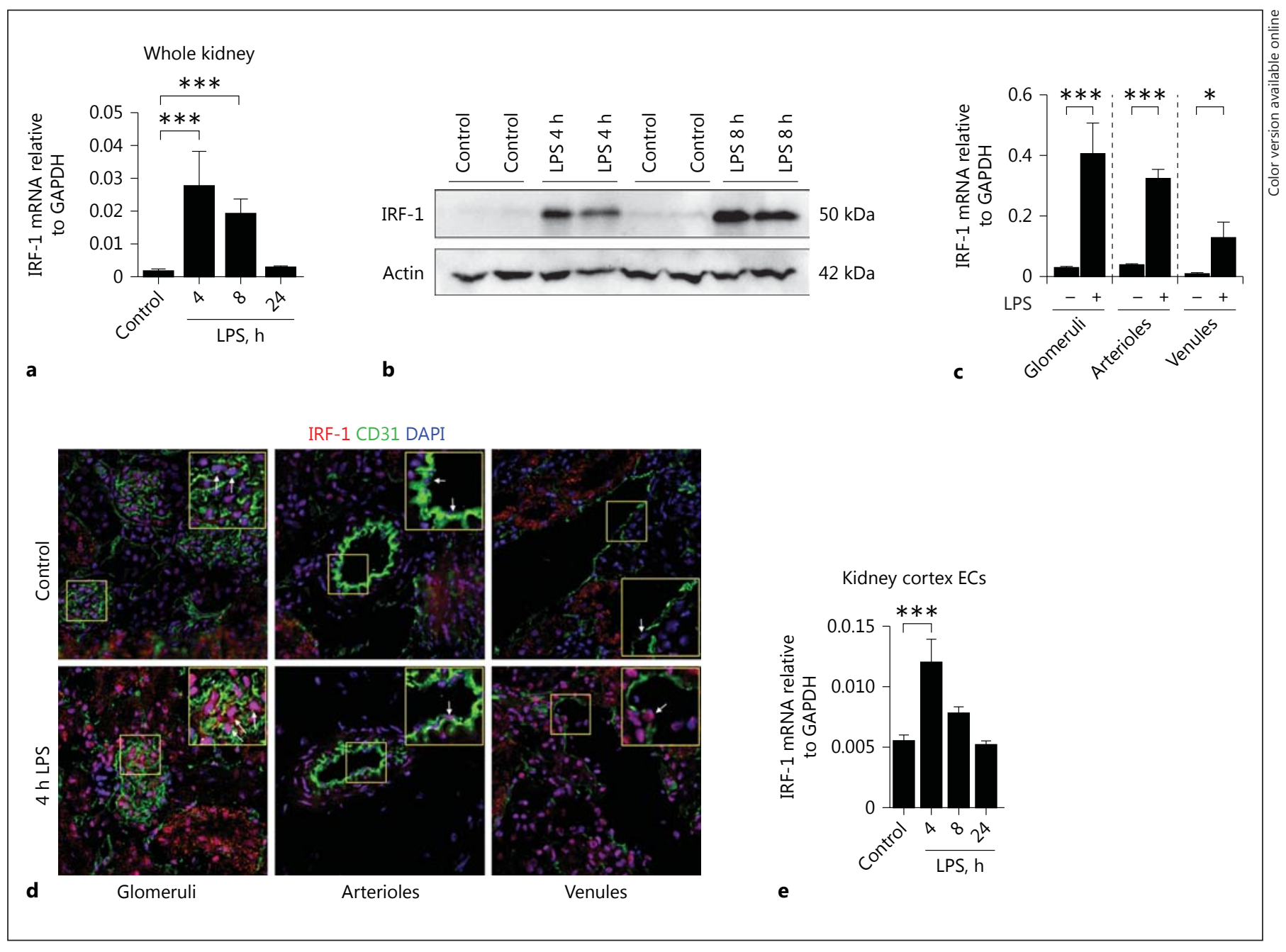

Fig. 1. IRF-1 is upregulated in the renal microvasculature of LPStreated mice. a Renal mRNA expression of IRF-1 in control and LPS-challenged ( $0.5 \mathrm{mg} / \mathrm{kg}$ i.p.) mice for the time points indicated. Bars represent the mean \pm SD of 5 mice per group. $\mathbf{b}$ Representative immunoblot of IRF-1 protein levels determined in whole kidney extracts of control (vehicle treated) mice and mice challenged with LPS ( $1 \mathrm{mg} / \mathrm{kg}$, i.p.) and sacrificed after 4 and $8 \mathrm{~h}$, respectively. Actin was used as a reference for protein loading. c mRNA expression levels of IRF-1 in renal microvascular compartments that were laser microdissected from kidneys of vehicle-treated (-) mice, and mice sacrificed $4 \mathrm{~h}$ after LPS administration (+). Bars represent the mean \pm SD of 3 mice per group. d Immunofluores-

ing $5 \%(\mathrm{v} / \mathrm{v})$ fetal calf serum. After washing, sections were incubated with donkey anti-rabbit antibody conjugated with Alexa Fluor ${ }^{\circledR} 555$-conjugated (A-31572), or goat anti-rat antibody conjugated with Alexa Fluor ${ }^{\circledR} 488$ (A-11006, both from Life Technologies) in $5 \%(\mathrm{v} / \mathrm{v})$ fetal calf serum $/ 2 \%(\mathrm{v} / \mathrm{v})$ normal mouse serum (Sanquin, Amsterdam, The Netherlands) in PBS for $45 \mathrm{~min}$. After washing, sections were incubated with $70 \%$ ethanol containing $0.1 \%(\mathrm{w} / \mathrm{v})$ Sudan Black B (Sigma-Aldrich) for $30 \mathrm{~min}$. Sections cence staining of endothelial marker CD31 (green), IRF-1 (red), and DAPI nuclear staining (blue) in the kidney of control mice (vehicle treated) and mice challenged with LPS (1 mg/kg, i.p.) and sacrificed $4 \mathrm{~h}$ later. All images were taken with equal exposure times. Original magnification $\times 400$. e IRF- 1 mRNA expression in primary mouse renal cortex endothelial cells (ECs) stimulated with LPS $(1 \mu \mathrm{g} / \mathrm{mL})$ or vehicle (control) for the indicated time periods. Bars represent the mean \pm SD of 4 samples and are representative of 2 independent experiments. All mRNA expression levels were determined by RT-qPCR using GAPDH as the housekeeping gene. Bars represent the mean \pm SD. ${ }^{*} p<0.05,{ }^{* * *} p<0.001$.

were mounted in Aqua/Polymount medium containing DAPI (1.5 $\mu \mathrm{g} / \mathrm{mL}$ ) (Polysciences, Warrington, PA, USA). Staining isotype controls included rat IgG2a (CN220020, Antigenix America Inc., Huntington Station, NY, USA) and rabbit IgG-UNLB (0111-01, Southern Biotech, Birmingham, AL, USA). Nonspecific secondary binding controls were performed as well. All control stainings were found to be devoid of staining (data not shown). Images were taken using a Leica DM 2000 fluorescence microscope (Leica 
Microsystems) equipped with a Nuance FX multispectral camera (Perkin Elmer, Groningen, The Netherlands). Image cubes were recorded using appropriate filters. Peak emission for Alexa Fluor 488 (CD31) was at $550 \mathrm{~nm}$ and for Alexa Fluor 555 (IRF-1) it was at $610 \mathrm{~nm}$. Image cubes were unmixed using Nuance software (version 3.0.1). Briefly, image unmixing allows subtraction of autofluorescence from and rendition of pure spectra in fluorescence photomicrographs, and thus crisp rendition of staining signal.

\section{Endothelial-Leukocyte Adhesion Assay}

HUVEC monolayers were transfected with either IRF-1 or scrambled siRNA. Forty-eight hours after transfection cells were treated with LPS (final concentration: $1 \mu \mathrm{g} / \mathrm{mL}$ ) for $4 \mathrm{~h}$. HL-60 leukocytes or THP-1 monocytic cells were labeled with Hoechst 33342 (Life Technologies) for $10 \mathrm{~min}$, washed and resuspended in RPMI/1\% (v/v) FBS (Sigma-Aldrich). Viability of the labeled HL60 and THP-1 cells was assessed by examining the FACS forward/side scatter plots, and always $>90 \%$. The labeled HL60 or THP- 1 cells were added to the HUVEC monolayers and incubated for $1 \mathrm{~h}$. After extensive washing, the supernatant containing nonadherent HL60 or THP-1 cells was removed and the endothelial cells plus adherent leukocytes or monocytes were trypsinized and resuspended in RPMI/1\% (v/v) FBS. The number of adhering cells was determined by flow cytometry using a MACSQuant Analyser (Miltenyi Biotech, Leiden, The Netherlands) according to the manufacturer's instructions.

Quantification of Cytokines IL-6 and IL-8 by ELISA

IL- 6 and IL- 8 concentrations were determined in the supernatants of HUVEC using either IL-6 and IL-8 Duo-Sets (R\&D Systems, Oxon, UK) or IL-6 and IL-8 MAX ${ }^{\mathrm{TM}}$ ELISA kits (BioLegend, San Diego, CA, USA), according to the manufacturer's protocol.

\section{Statistical Analysis}

Statistical analysis of results was performed by a 2-tailed unpaired Student $t$ test, assuming equal variances to compare 2 replicate means, or by 1-way ANOVA followed by Bonferroni post hoc correction to compare multiple replicate means. All statistical analyses were performed using GraphPad Prism Software v.7 (GraphPad Prism Software Inc., San Diego, CA, USA). Differences were considered significant when $p<0.05$.

\section{Results}

IRF-1 Is Upregulated in the Renal Microvasculature of LPS-Treated Mice

To determine whether IRF-1 was regulated by LPS in vivo, we initially investigated whether IRF-1 was expressed in the kidneys of healthy and LPS-treated mice. We found low expression of IRF-1 in healthy mouse kidney, whereas LPS administration resulted in a rapid increase of IRF-1 mRNA and protein levels in whole kidney tissue lysates (Fig. 1a, b). Analysis of renal microvascular segments revealed that IRF-1 mRNA was upregulated in all 3 microvascular compartments of the mouse kidney in response to LPS (Fig. 1c). IRF-1 nuclear localization was found in glomerular cells, in arteriolar endothelial cells, and to a lesser extent in the venules of LPS-treated mice (Fig. 1d). The extent of endothelial nuclear IRF-1 localization, especially in glomeruli and arterioles, was more pronounced in mice that were treated with LPS and sacrificed at $4 \mathrm{~h}$ after administration than in mice sacrificed at $8 \mathrm{~h}$ after LPS administration (Fig. 1d, see online suppl. Fig. 2a). Additionally, IRF-1 nuclear localization was also found in some, but not all, tubular epithelial cells in LPStreated mice (see online suppl. Fig. 2b), supporting earlier observations [18]. We also found a temporal increase in IRF-1 mRNA levels in primary endothelial cells isolated from the mouse kidney cortex and exposed to LPS in vitro (Fig. 1e). From these data, we conclude that IRF-1 is constitutively expressed in all of the different renal microvascular compartments and tubular epithelial cells and that its expression is upregulated by LPS.

\section{LPS Induces IRF-1 Upregulation and Nuclear \\ Translocation in Human Endothelial Cells}

We wanted to further investigate the mechanisms of IRF-1 regulation and the consequences of IRF-1 depletion, and decided to proceed with HUVEC for these mechanistic studies as primary renal microvascular endothelial cells are difficult to isolate and culture in sufficient numbers for extensive molecular studies. HUVEC provide a classic model system to study many different aspects of endothelial function, the use of which is widely accepted in the field. Moreover, our initial finding using mouse kidney cortex endothelial cells that IRF-1 mRNA and protein levels were upregulated as a result of LPS exposure were recapitulated in HUVEC (Fig. 2a-c). Examining the kinetics of IRF-1 nuclear translocation after LPS stimulation in HUVEC revealed that at $1 \mathrm{~h}$ after LPS administration IRF-1 protein became visible in the cytoplasm and at $2 \mathrm{~h}$ had started to translocate from the cytoplasm to the nucleus. Translocation reached a maximum at $4 \mathrm{~h}$ after LPS administration and thereafter diminished (Fig. 2d, e).

\section{IRF-1 Regulates LPS-Mediated VCAM-1 Expression}

We proceeded to investigate whether there was a role for IRF- 1 as a regulator of LPS-induced expression of endothelial adhesion molecules. siRNA-based IRF-1 knockdown in HUVEC did not affect LPS-induced upregulation of the endothelial adhesion molecules E-selectin and ICAM-1, nor the induction of the inflammatory cytokines IL-6 and IL-8 (Fig. 3a-c). In contrast, the upregulation of VCAM-1 was significantly reduced, although not completely inhibited, in the absence of IRF-1 (Fig. 3a-c). 


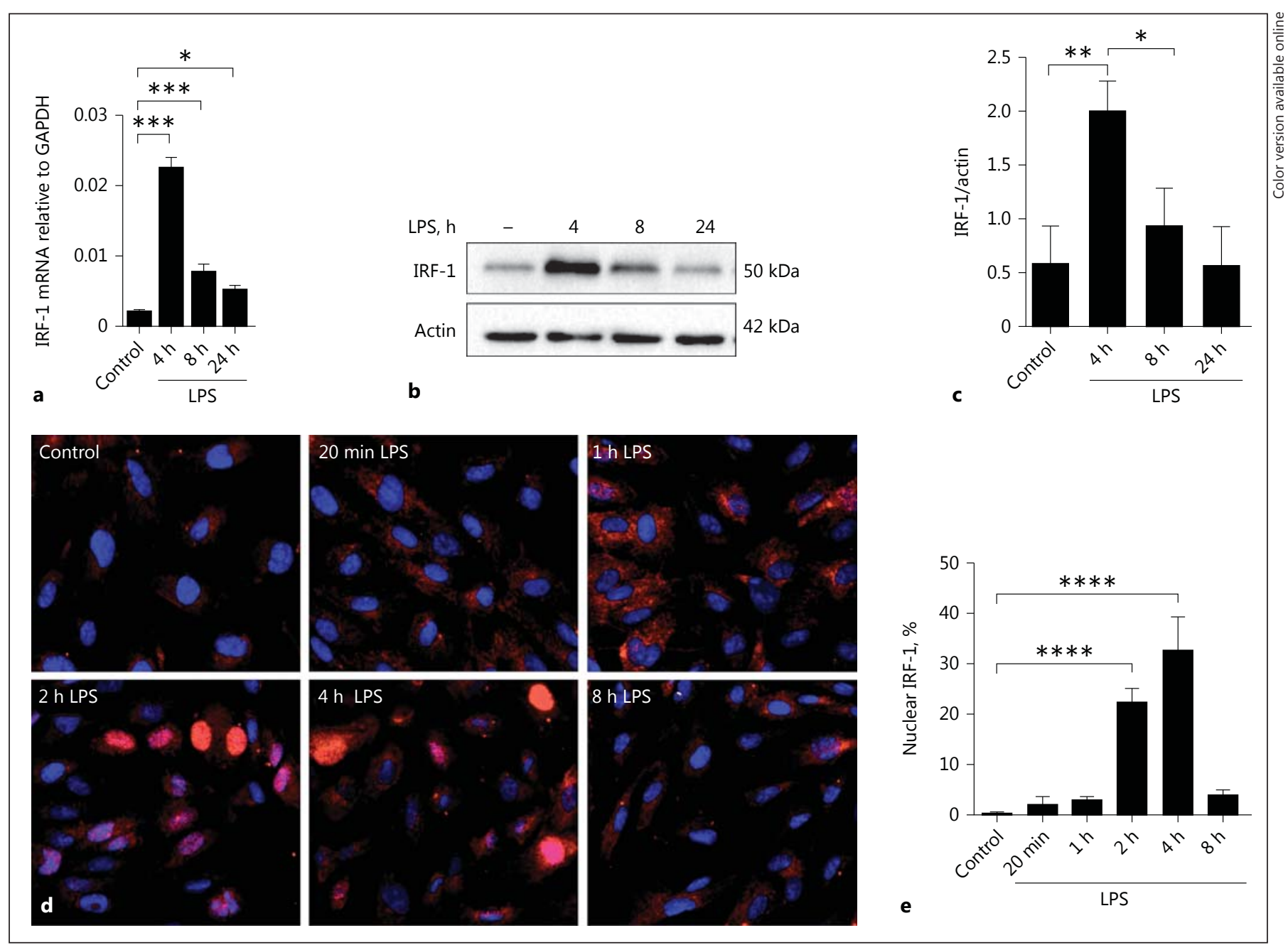

Fig. 2. LPS induces IRF-1 upregulation and nuclear translocation in human endothelial cells. a Kinetics of IRF-1 mRNA expression in HUVEC upon treatment with vehicle (control) or LPS for the indicated time periods as determined by RT-qPCR using GAPDH as the housekeeping gene. Data are shown as means \pm SD of 4 samples from one experiment and are representative of 3 independent experiments. ${ }^{*} p<0.05,{ }^{* * *} p<0.001$. b Whole cell lysates of HUVEC treated with vehicle $(-)$ or LPS $(1 \mu \mathrm{g} / \mathrm{mL})$ for the indicated time periods were subjected to immunoblot analysis. Actin was used as a reference for protein loading. The immunoblots shown are representative of blots from 3 independent experi-

Fig. 3. IRF-1 regulates LPS-mediated VCAM-1 expression. a Gene expression levels of IRF-1, E-selectin, VCAM-1, ICAM-1, IL-6, and IL- 8 were determined by RT-qPCR in HUVEC transfected with scrambled (siScr) or IRF-1 (siIRF-1) specific siRNA and subsequently treated with vehicle (control) or LPS for $4 \mathrm{~h}$. GAPDH was used as the housekeeping gene. Bars represent the mean \pm SD of 4 samples from one experiment and are representative of 3 independent experiments. ${ }^{* * *} p<0.001$. b Whole cell lysates from siScr- and siIRF-1-transfected HUVEC exposed to vehicle (-) or LPS for $4 \mathrm{~h}$ were subjected to immunoblot analysis for IRF-1,

Endothelial IRF-1 Regulates LPS-Induced VCAM-1 Induction ments. c Densitometric analysis of IRF-1 protein bands as described in Materials and Methods. Bars represent the mean $\pm \mathrm{SD}$ of 3 independent experiments. ${ }^{*} p<0.05,{ }^{* *} p<0.01$. d Immunofluorescence images show staining of IRF-1 (red) and DAPI nuclear staining (blue) in HUVEC treated with vehicle or LPS at different time points. Data show representative images of 3 independent experiments. e The percentage of cells with IRF-1 localized in the nucleus was quantified, at least 350 cells from each sample were analyzed. All images were taken with equal exposure times, original magnification $\times 400$. Bars represent the mean \pm SD of 3 independent experiments. ${ }^{* * * *} p<0.0001$.

VCAM-1, E-selectin, and ICAM-1 protein expression using actin as a reference for protein loading. The immunoblots shown are representative of blots from 3 independent experiments. c Densitometric analysis of protein bands as described in Materials and Methods. Bars represent the mean \pm SD of 3 independent experiments. ${ }^{* * *} p<0.0001$. d The percentage of HL60 and THP- 1 cells that adhered to confluent monolayers of siScr- and siIRF-1-transfected HUVEC treated with vehicle (control) or LPS for $4 \mathrm{~h}$. Bars represent the mean \pm SD of 3 samples from one experiment and are representative of 3 independent experiments.

(For figure see next page.)

J Innate Immun 2017;9:546-560

DOI: $10.1159 / 000477211$
551 


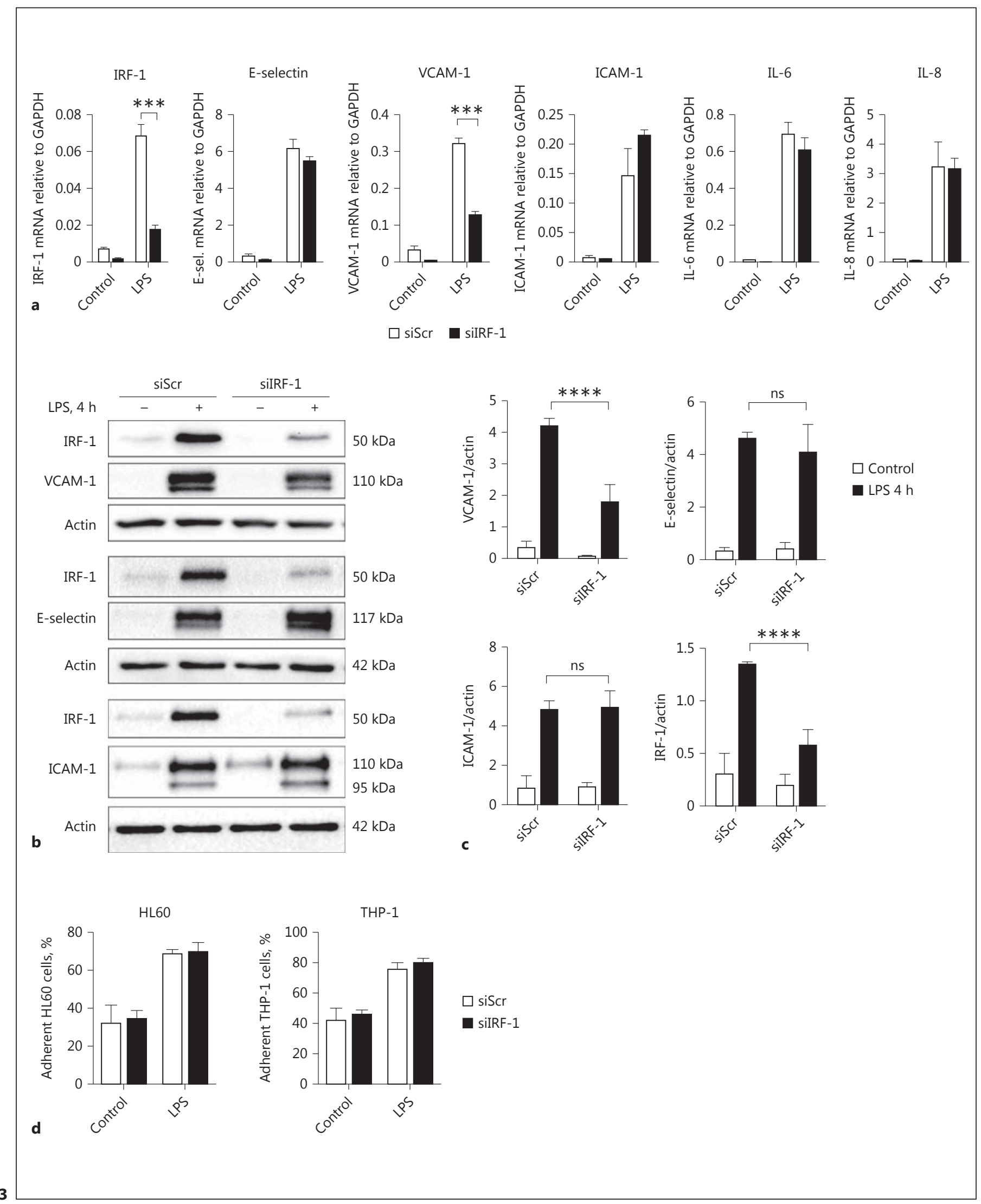



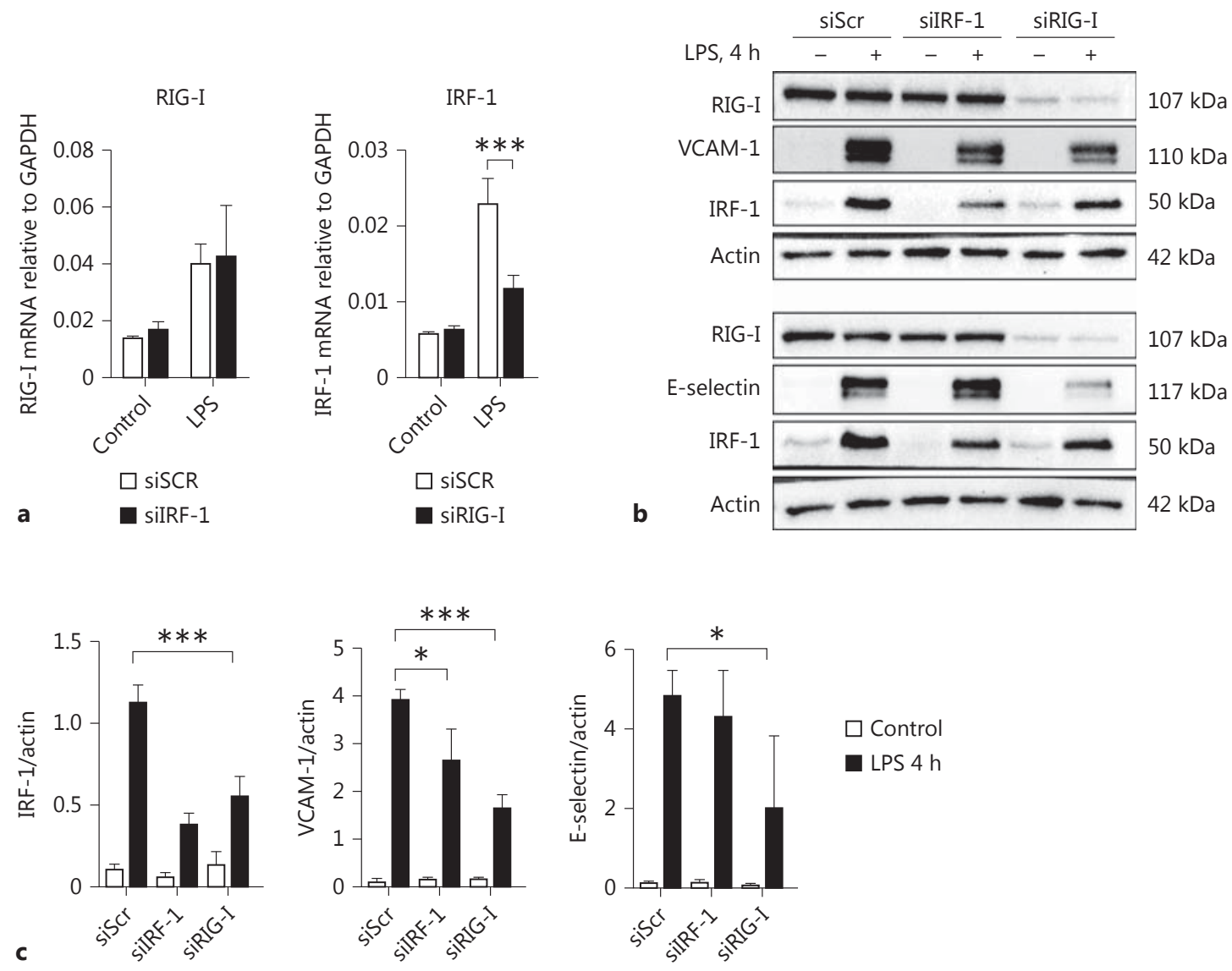

Fig. 4. RIG-I regulates VCAM-1 expression in part by controlling LPS-mediated induction of IRF-1. a RIG-I and IRF-1 mRNA levels were assessed by RT-qPCR in HUVEC transfected with siScr, siIRF-1, or siRIG-I and subsequently treated with vehicle (control) or LPS for $4 \mathrm{~h}$, using GAPDH as a housekeeping gene. Bars represent the mean \pm SD of 4 samples from one experiment and are representative of 3 independent experiments. ${ }^{* * *} p<0.001$. b RIG-I, IRF-1, VCAM-1, and E-selectin protein expression in

The functional consequences of IRF-1 knockdown were studied by determining adhesion of HL60 leukocytic cells and THP-1 monocytic cells to activated HUVEC after LPS treatment. Despite reduced VCAM-1 expression in IRF-1-deficient cells, we found no effect on the number of HL60 or THP-1 cells adhering to LPS-stimulated HUVEC when compared to control cells (Fig. 3d). Therefore, these results suggest a role for IRF-1 as a regulator of VCAM-1 induction in response to LPS treatment, without a concurrent effect on leukocyte adhesion to the endothelium under the conditions studied.

Endothelial IRF-1 Regulates LPS-Induced VCAM-1 Induction whole cell lysates from siScr, silRF-1, and siRIG-I transfected HUVEC after vehicle (control) or LPS treatment for $4 \mathrm{~h}$ was assayed by Western blot. Actin was used as a reference for protein loading. The Western blots are representative of 3 independent experiments. c Densitometric analysis of IRF-1, VCAM-1, and E-selectin protein bands as described in Materials and Methods. Bars represent the mean \pm SD of 3 independent experiments. ${ }^{*} p<$ $0.05,{ }^{* * *} p<0.001$.

\section{RIG-I Regulates VCAM-1 Expression in Part by Controlling LPS-Mediated Induction of IRF-1}

RIG-I acts as an intracellular double-stranded RNA (dsRNA) receptor together with its adaptor protein mitochondrial antiviral signaling (MAVS). IRF-1 was shown to regulate basal transcription, as well as dsRNA-mediated upregulation of RIG-I [24]. Recent data from our laboratory showed that in endothelial cells, RIG-I is an intracellular receptor that regulates LPS-induced endothelial activation [13]. Based on these findings we hypothesized that IRF-1 was an upstream regulator of RIG-I- 


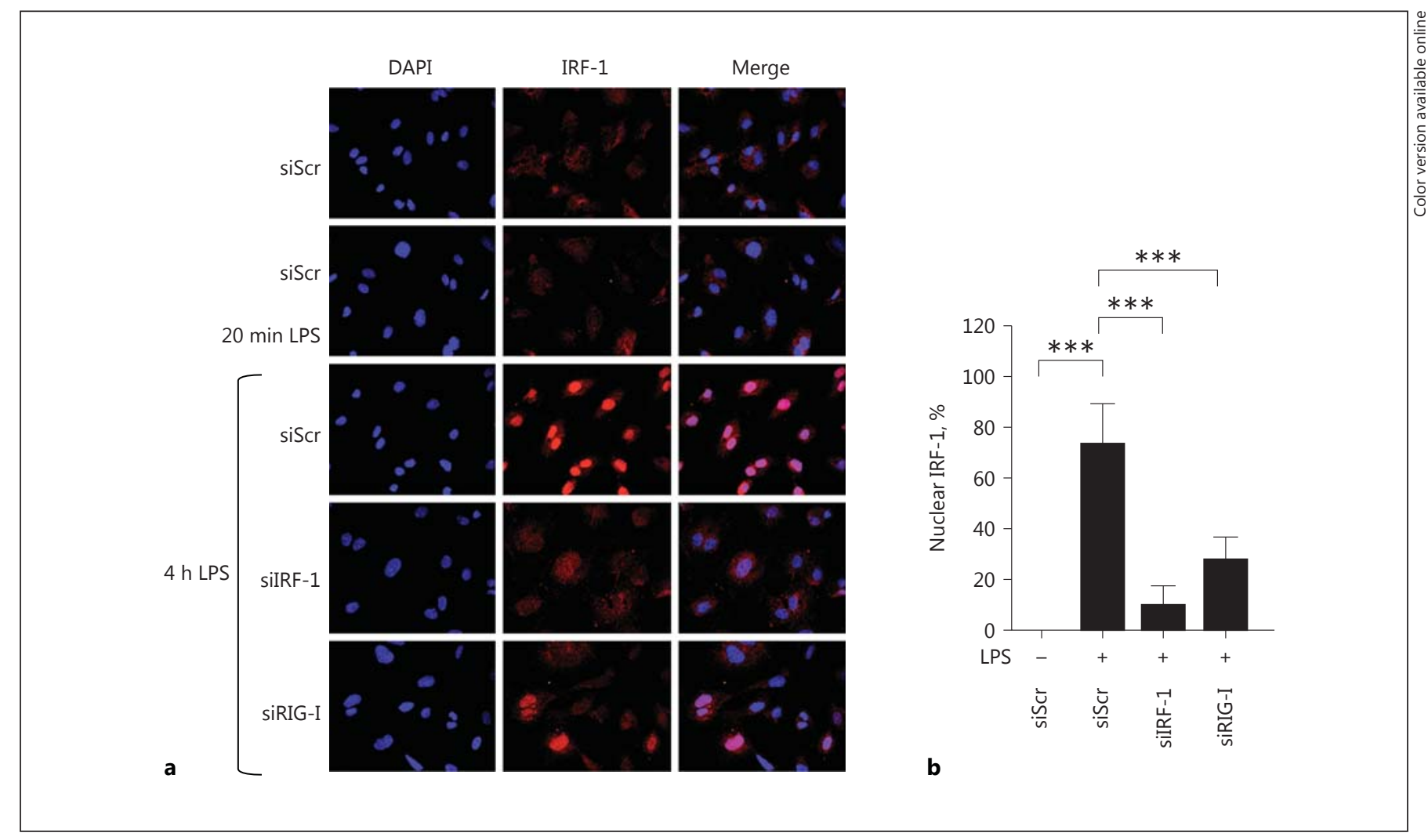

Fig. 5. LPS-mediated nuclear translocation of IRF-1 is dependent on RIG-I. a Representative immunofluorescence images showing IRF-1 (red) and DAPI nuclear staining (blue) in HUVEC transfected with either siScr, siIRF-1, or siRIG-I and subjected to vehicle (-), 20 min or $4 \mathrm{~h}$ LPS. All images were taken with equal exposure times. Original magnification $\times 400$. $\mathbf{b}$ The percentage of nuclear IRF-1 was quantified by analyzing at least 250 cells from each sample. Bars represent mean \pm SD and data are representative of 3 independent experiments. ${ }^{* * *} p<0.001$. mediated endothelial responses to LPS. Surprisingly, we found that the mRNA and protein levels of RIG-I were not affected in HUVEC lacking IRF-1 (Fig. 4a-c). In contrast, RIG-I knockdown partially inhibited LPS-mediated IRF-1 gene and protein induction (Fig. $4 \mathrm{a}-\mathrm{c}$ ). In addition, RIG-I knockdown inhibited LPS-mediated upregulation of VCAM-1 and E-selectin (Fig. 4b, c), confirming previous findings [13], whereas IRF-1 knockdown only inhibited LPS-mediated VCAM-1 induction (Fig. 3b, 4b). From these findings it can be concluded that IRF-1 does not regulate LPS-driven RIG-I expression. In contrast, RIG-I is an upstream regulator of LPS-driven IRF-1 expression that specifically regulates VCAM-1 induction.

\section{LPS-Mediated Nuclear Translocation of IRF-1 Is \\ Dependent on RIG-I}

We next investigated whether nuclear translocation of IRF-1 was dependent on RIG-I using RIG-I knockdown in HUVEC. Consistent with our previous results, IRF-1 translocated to the nucleus within $4 \mathrm{~h}$ of LPS activation in siScr-treated cells. RIG-I knockdown effectively inhibited IRF-1 translocation upon LPS stimulation (Fig. 5a, b), further corroborating that RIG-I functions as an upstream regulator of LPS-induced IRF-1 expression and nuclear localization.

\section{LPS-Mediated Endothelial Activation Is Regulated by \\ a Yet Unknown Mechanism in the Absence of Both \\ IRF-1 and MAVS}

Recent findings from our laboratory have shown that the RIG-I adaptor protein MAVS also regulates LPSinduced endothelial activation via the NFKB signaling pathway [13]. Knockdown of MAVS partially inhibited VCAM-1 expression (Fig. 6a, b), confirming our previous findings [13]. To investigate whether IRF-1 and MAVS are part of the same pathway or rather represent different LPS-controlled signaling pathways in endothelial cells, we knocked down IRF-1, MAVS, or IRF-1 and MAVS DOI: $10.1159 / 000477211$
Yan et al. 


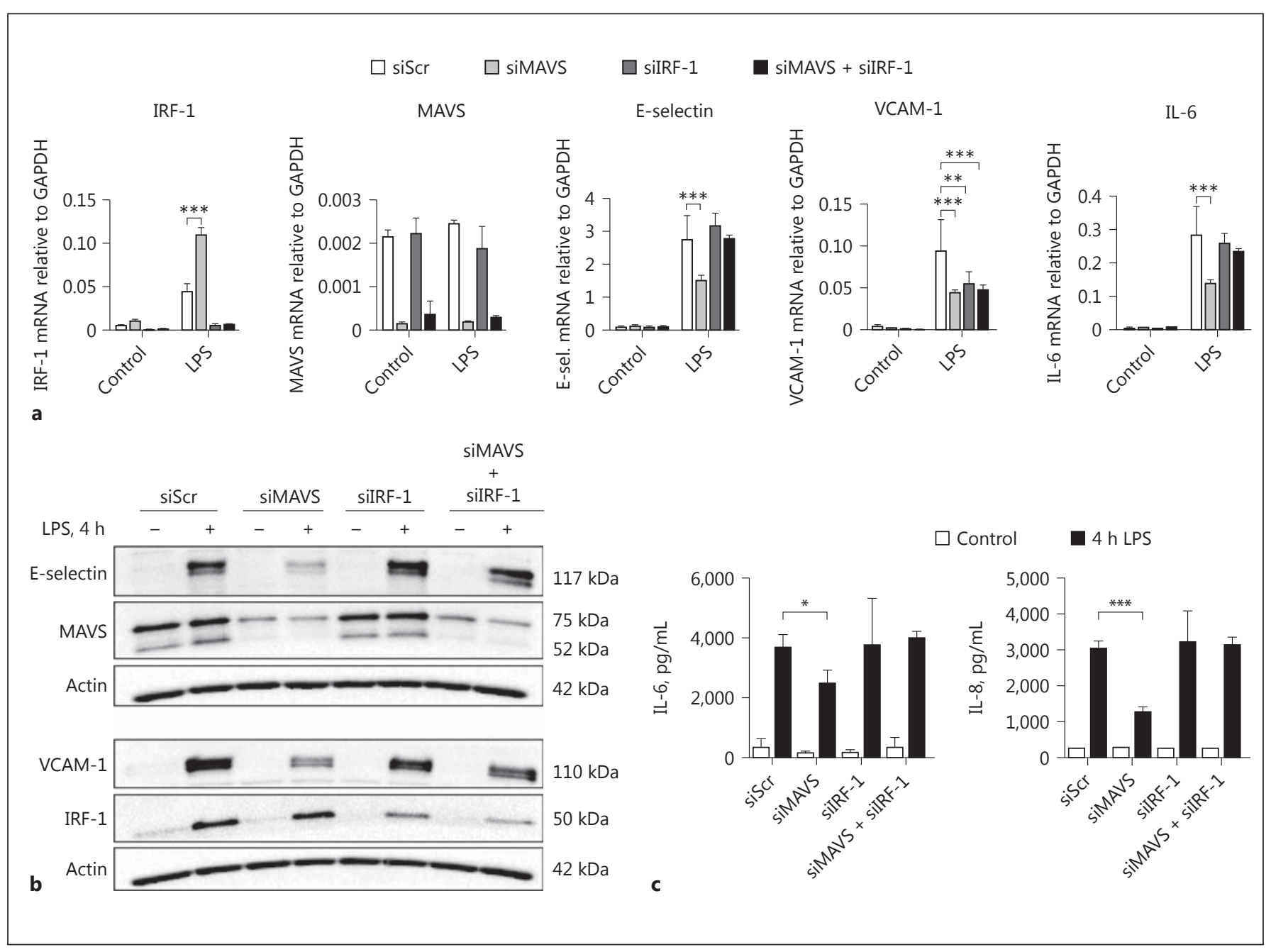

Fig. 6. In the absence of both IRF-1 and MAVS, LPS-mediated endothelial activation is regulated by an unknown mechanism. a Gene expression levels of IRF-1, MAVS, E-selectin, VCAM-1 and IL- 6 were assessed by RT-qPCR in HUVEC transfected with siScr, siMAVS, siIRF-1, or both siMAVS, and siIRF-1, and treated with vehicle (control) or LPS for $4 \mathrm{~h}$, using GAPDH as the housekeeping gene. Bars represent the mean \pm SD of 4 samples from one experiment and data are representative of 3 independent experiments. ${ }^{* *} p<0.01,{ }^{* * *} p<0.001$. b E-selectin, MAVS, VCAM-1, and IRF-1 protein expression in HUVEC transfected with siScr, siMAVS, siIRF-1, or double knockdown of siMAVS and siIRF-1,

simultaneously, prior to LPS exposure. In IRF-1-deficient HUVEC, only LPS-mediated VCAM-1 induction was inhibited (Fig. 6a). In contrast, in the absence of MAVS, the upregulation of the endothelial adhesion molecules E-selectin, VCAM-1, and the inflammatory cytokine IL-6 were all significantly inhibited (Fig. 6a). Finally, in double-knockdown cells, VCAM-1 expression was inhibited to a similar level as in IRF-1 or MAVS-

Endothelial IRF-1 Regulates LPS-Induced

VCAM-1 Induction after $4 \mathrm{~h}$ of vehicle treatment (-) or LPS stimulation as assessed by immunoblotting of whole cell extracts. Actin was used as a reference for protein loading. The immunoblots depicted are representative of 3 independent experiments. c IL- 6 and IL-8 cytokine concentrations in the culture medium from siScr-, siMAVS-, siIRF-1-, or siMAVS- and siIRF-1-transfected HUVEC exposed to vehicle (control) or LPS for $4 \mathrm{~h}$ were determined by ELISA. Bars represent the mean \pm SD of 4 samples from one experiment and data are representative of 3 independent experiments. ${ }^{*} p<0.05,{ }^{* * *} p<$ 0.001 .

deficient conditions (Fig. 6a). Since VCAM-1 induction in endothelial cells lacking both IRF-1 and MAVS was not additionally inhibited, IRF-1 and MAVS probably reside in the same signaling pathway. Unexpectedly, we found that E-selectin and IL-6 induction in IRF-1 and MAVS double-knockdown cells was no longer inhibited when compared to MAVS-only deficient cells. Assessment of protein levels by Western blot for E-selectin, 
VCAM-1, and by ELISA for cytokines IL- 6 and IL- 8 revealed similar results (Fig. 6b, c). Using RT-qPCR we showed that TLR4 mRNA levels were increased 1.5-fold in the absence of either IRF-1 or MAVS, as well as in double IRF-1 and MAVS knockdown HUVEC, compared to control HUVEC (data not shown). From these findings we conclude that LPS-mediated endothelial activation is regulated by an as yet unknown mechanism in the absence of IRF- 1 and MAVS.

\section{IRF-1 Relays LPS-Mediated Proinflammatory}

Signaling Independent of $N F \kappa B$

Several studies have shown that NFKB activation plays a key role in the pathogenesis of sepsis [11]. In addition, $\mathrm{NF \kappa B}$ is a critical regulator of endothelial inflammatory responses, including VCAM-1 regulation [25]. To address whether a possible connection exists between IRF-1 and NFKB signaling in the regulation of VCAM-1, we examined whether $\mathrm{NF \kappa B}$ signaling was dependent on IRF-1. In LPS-treated HUVEC previously transfected

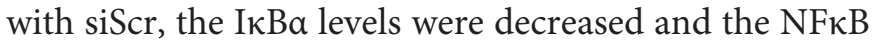
p65 subunit translocated to the nucleus (Fig. 7a-d). IRF-1 depletion did not affect LPS-induced IкBa degradation or NFkB p65 nuclear translocation (Fig. 7a-d). These observations indicate that IRF-1 regulates LPS-mediated VCAM-1 expression independent of NFKB involvement.

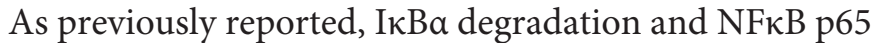
nuclear translocation were inhibited in MAVS-deficient endothelial cells [13] (Fig. 7a-d). In contrast to MAVS single knockdown, we show that in MAVS and IRF-1 double-knockdown HUVEC treated with LPS, IкBa was degraded, which was paralleled by NFkB p65 nuclear translocation (Fig. 7a, b). These results reveal that in the absence of both MAVS and IRF-1, an NFkB-driven pathway mediates endothelial activation, and that the protective effect of MAVS deficiency on I $\mathrm{K} B \alpha$ degradation and $\mathrm{NF \kappa B}$ translocation to the nucleus is lost in the absence of IRF-1.

\section{Discussion}

Sepsis is a life-threatening condition caused by a dysregulated host response to infection and a leading cause of mortality in critically ill patients worldwide [3]. The pathogenesis of sepsis is complicated and controlled by many different biological factors and regulatory mechanisms, of which the majority is still unknown. Microvascular endothelial changes leading to disturbances in blood flow, vascular leakage, and exaggerated endothe- lial activation are known to promote organ failure in sepsis [1]. As a consequence of this lack of understanding, targeted molecular interventions in sepsis have been unsuccessful. To find new potential microvascular targets in sepsis, the current study aimed to explore the role of IRF-1 in LPS-induced endothelial proinflammatory activation. Our findings reveal that IRF-1 is responsible for LPS-mediated VCAM-1 induction, but does not play a role in the regulation of E-selectin and ICAM-1, or the proinflammatory cytokines IL-6 and IL-8. Moreover, IRF-1 regulates VCAM-1 induction by a mechanism independent of NFKB. We found RIG-I to be an upstream regulator of IRF-1 transcriptional responses to LPS. Taken together, our data reveal that upon LPS stimulation, a presently undefined RIG-I-mediated, IRF-1-based, NFKB-independent, proinflammatory pathway is activated that specifically controls VCAM-1 expression.

In this study, we identified a role for IRF- 1 as a regulator of VCAM-1 induction by LPS. A role for endothelial IRF- 1 as a regulator of TNF- $\alpha$-mediated VCAM- 1 induction in endothelial cells was reported before [20,21]. Our data reveal that IRF-1 is expressed in mouse renal endothelial and tubular epithelial cells, and that LPS can induce renal IRF-1 expression in vivo. LPS-mediated IRF-1 induction occurred in all microvascular compartments. Wang et al. [21] reported similar findings in TNF- $\alpha-$ treated mice, where IRF-1 was found localized in the endothelium of the renal microvasculature and the aorta. The up- and downstream signaling pathways clearly differ between LPS and TNF- $\alpha$-activated endothelial cells. In primary mouse endothelial cells, TNFR2 activation by TNF- $\alpha$ was shown to induce IFN- $\beta$ signaling, thereby promoting monocyte recruitment through the induction of IRF-1 [20]. In HUVEC, we found low expression levels of IFN- $\beta$ and no significant increase in expression when stimulated with LPS (data not shown). Hence, LPS-mediated induction of IRF- 1 is not a result of autocrine IFN- $\beta$ expression.

Previous work from our laboratory identified RIG-I and TLR4 as 2 independent receptors required for the regulation of endothelial activation in response to LPS [13]. We hypothesized that IRF-1 would act as an upstream regulator of RIG-I-mediated endothelial responses to LPS, based on the observation reported previously that IRF-1 regulated basal RIG-I expression and the dsRNA-mediated induction of RIG-I in human immortalized epithelial cells [24]. Additionally, IRF-1 was required to mediate the induction of RIG-I by 25 -hydroxycholesterol in HUVEC [26], and the ataxia-telangiectasia
556

J Innate Immun 2017;9:546-560 DOI: $10.1159 / 000477211$
Yan et al. 


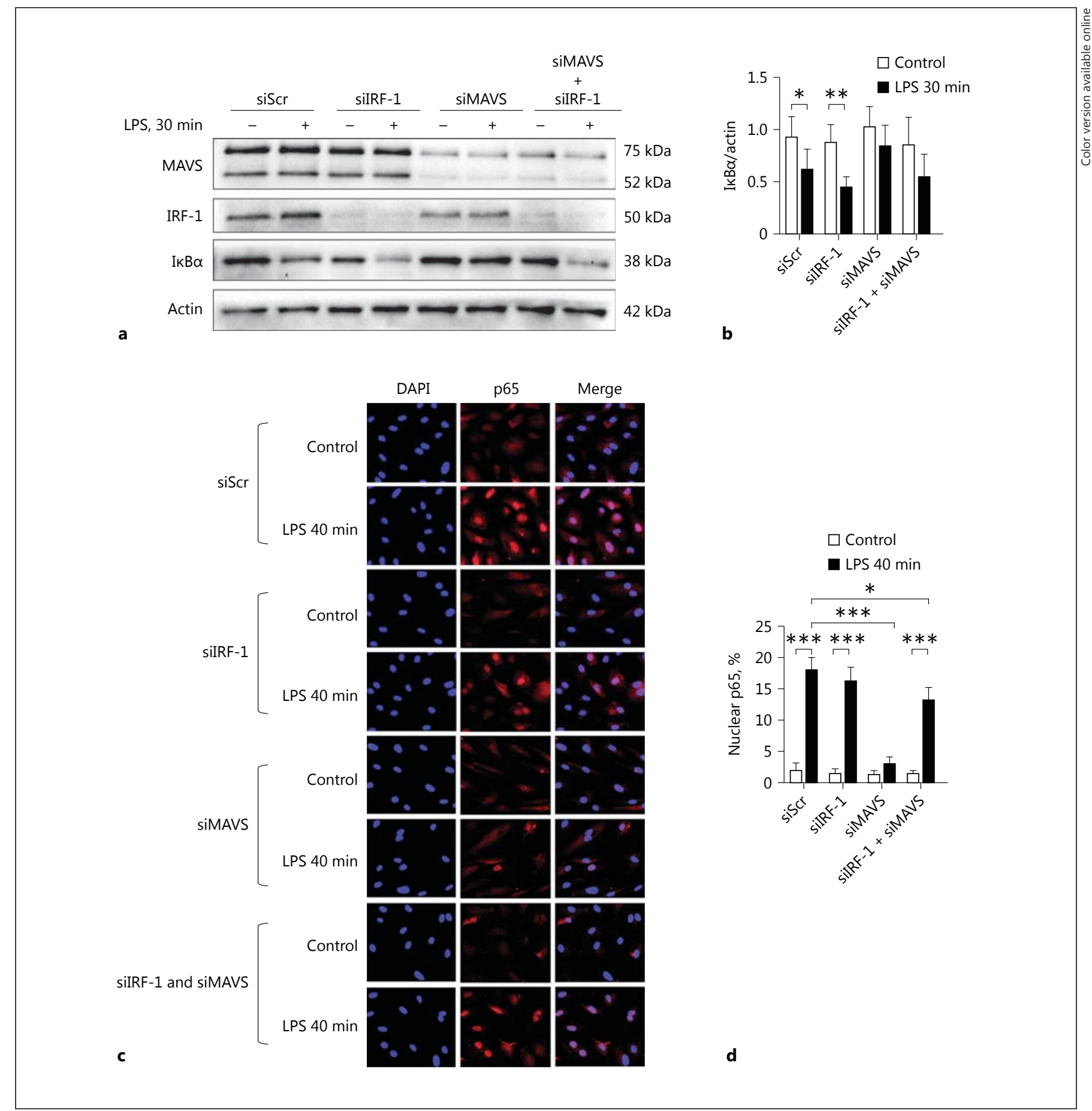

Fig. 7. IRF-1 relays LPS-mediated proinflammatory signaling independent of NFKB. a Immunoblot analysis of MAVS, IRF-1, and $\mathrm{I} \kappa \mathrm{B} a$ protein levels in whole cell lysates from HUVEC transfected with different siRNAs and treated with vehicle (-) or LPS for 30 $\mathrm{min}$. Actin was used as a reference for protein loading. Representative immunoblots of 3 independent experiments are shown. b Densitometric analysis of protein bands as described in Materials and Methods. Bars represent the mean \pm SD of 3 independent experiments. ${ }^{*} p<0.05,{ }^{* *} p<0.01$. c HUVEC transfected with
siScr, siIRF-1, siMAVS, or siMAVS and siIRF-1, and then treated with vehicle (-) or LPS for 40 min were subjected to immunostaining for p65 (Rel A) protein. All images were taken with equal exposure times; original magnification $\times 400$. d The percentage of nuclear NFKB p65 localization was quantified by analyzing at least 350 cells from each sample. Bars represent the mean \pm SD and data are representative of 3 independent experiments. ${ }^{*} p<0.05$, $* * * p<0.001$. 
mutated-IRF-1 signaling pathway regulated RIG-I mediated endothelial activation associated with endothelial senescence [27]. However, our current data imply that in the case of LPS stimulation, RIG-I is an upstream regulator of IRF-1-mediated inflammatory responses. The regulation of endothelial RIG-I-IRF-1 signaling thus seems to depend on the nature of the activating stimuli and the concurrent signaling pathways activated.

VCAM-1 induction by LPS was partly inhibited in IRF-1-deficient cells, while the expression of E-selectin, ICAM-1, IL-6, and IL-8 remained unaffected. Previous studies found that NFKB is a main but not solely responsible driver of endothelial activation. Other transcription factors are required to assemble with $\mathrm{NF \kappa B}$ to generate the transcriptional activating complexes needed for the induction of the expression of endothelial adhesion molecules [28]. LPS was found to induce endothelial activation by the activity of several molecules such as NFKB, activator protein 1 (AP-1), specificity protein 1 (SP-1), IRF-1, and GATA-2. However, unlike the VCAM-1 promoter which has functional binding sites for all of these transcription factors, no functional binding sites for IRF-1 were found in the E-selectin and ICAM-1 promoters [28-30]. This may explain why we only observed an inhibitory effect of IRF-1 depletion on VCAM-1 induction upon exposure of HUVEC to LPS. Moreover, the fact that IRF-1 knockdown did not completely inhibit LPSmediated VCAM-1 expression may be due to additional unknown VCAM-1 regulating pathways that are activated by LPS.

Our results establish a role for IRF-1 as a regulator of VCAM-1 induction by LPS, which we show to be independent of NFkB. Similar findings were observed by Dagia et al. [30] who found that TNF- $\alpha$ activation of endothelial cells results in IRF-1-mediated VCAM-1 expression through binding of IRF-1 to the VCAM-1 promotor. Similarly, DeVerse et al. [31] showed that in cultured endothelial cells, fluid shear stress signaling, synergistic with postprandial triglyceride-rich lipoproteins, transcriptionally augments cytokine-induced VCAM-1 expression in an IRF-1-dependent manner. In addition, Warfel and Agnillo [32] reported that anthrax lethal toxin enhanced nuclear expression of endothelial IRF-1 and induced VCAM-1 expression. Lethal toxin-mediated enhancement of TNF- $\alpha$-induced VCAM-1 expression was found to be transcriptionally driven by the cooperation of both increased NFKB and STAT1 activation, promoting IRF-1 expression, which then mediates VCAM-1 induction. Likewise, cytokine-driven NFKB binding to the binding motif of the VCAM-1 promoter was shown to enhance the binding of IRF-1 to its cognate site [28]. Hence, our data and that of others suggest that depending on the activating stimuli, IRF-1 in combination with NFKB mediates TNF- $\alpha$-mediated induction of VCAM-1.

In addition to these findings, leukocytic or monocytic cell adhesion to the LPS-activated endothelium was not disrupted by IRF-1 deficiency, despite diminished VCAM-1 induction. This suggests that the residual VCAM-1 was still sufficient to allow leukocyte adhesion and/or that other IRF-1-VCAM-1 independent factors, such as the nonaffected E-selectin and cytokine expression, may be required for the regulation of leukocyte adhesion to the endothelium. Indeed, adhesion of HL-60 cells to the endothelium was found to be primarily driven by E-selectin upon TNF- $\alpha$ stimulation [33]. Both this latter study and our study were performed under static conditions. Previous studies found monocyte adhesion to TNF- $\alpha$-treated endothelium to be regulated by IRF-1mediated VCAM-1 induction under flow conditions [20, $30,31]$. In patients with septic shock, increased endothelial permeability and vasodilation result in a dramatic drop in blood flow rate in certain microvascular compartments such as the capillaries $[6,7,9]$. Our findings under static conditions may suggest that inhibiting endothelial IRF-1 in patients with near-static blood flow may not result in an inhibitory effect on leukocyte adhesion to the activated endothelium, despite reduced VCAM-1 expression levels. Nevertheless, future studies investigating whether IRF-1 also mediates leukocyte adhesion to LPSactivated endothelium under high- and low-flow conditions will provide important additional insights into these functional consequence matters of IRF-1 blockade/absence.

We hypothesized that MAVS and IRF-1 may be separate signaling pathways induced by LPS to regulate endothelial activation. Surprisingly, double knockdown of both IRF-1 and MAVS did not result in additional inhibition of VCAM-1 induction, suggesting that MAVS and IRF-1 reside in the same signaling pathway. In immune cells, peroxisomal MAVS was shown to activate IRF-1, leading to rapid expression of antiviral genes triggering an immediate IFN-independent antiviral response [34]. In endothelial cells, a similar pathway mediating IFNindependent endothelial activation by LPS likely exists. We also found that upregulation of E-selectin and IL-6 was not affected by both MAVS and IRF-1 deficiency, suggesting that in the absence of both of these factors an as yet undefined NFKB-driven pathway takes over to mediate induction of E-selectin and IL-6. Preliminary data
558

J Innate Immun 2017;9:546-560 DOI: $10.1159 / 000477211$
Yan et al. 
on alternative signaling pathways becoming more or less activated in the absence of IRF-1 and MAVS identified altered MAPK and PI3K/AKT signaling (data not shown), which will be investigated in more detail in future studies using quantitative kinome profiling technology. These findings are important when considering IRF-1, MAVS, or other mediators of endothelial activation as targets for therapeutic intervention in sepsis since multiple redundant pathways are, amongst others, a cause of failed clinical trials directed at inhibiting a single factor or a selected part of the inflammatory pathway [35].
In summary, we have identified an IRF-1 signaling pathway in endothelial cells which is activated by LPS and specifically regulates VCAM-1 induction by a mechanism that is independent of NFKB. Moreover, we identified RIG-I to be an upstream regulator of IRF-1-mediated endothelial responses to LPS.

\section{Acknowledgements}

We would like to thank Henk E. Moorlag of the UMCG Endothelial Cell Facility for his excellent technical support. This work was supported by the research foundation of the Department of Critical Care, UMCG, and the China Scholarship Council (R.Y.).

\section{References}

1 Angus DC, van der Poll T: Severe sepsis and septic shock. N Engl J Med 2013;369:840-851.

2 Vincent JL, Opal SM, Marshall JC, Tracey KJ: Sepsis definitions: time for change. Lancet 2013;381:774-775.

3 Singer M, Deutschman CS, Seymour CW, Shankar-Hari M, Annane D, Bauer M, et al: The Third International Consensus Definitions for Sepsis and Septic Shock (Sepsis-3). JAMA 2016;315:801-810.

4 Angus DC, Linde-Zwirble WT, Lidicker J, Clermont G, Carcillo J, Pinsky MR: Epidemiology of severe sepsis in the United States: analysis of incidence, outcome, and associated costs of care. Crit Care Med 2001;29:13031310.

5 Martin GS: Sepsis, severe sepsis and septic shock: changes in incidence, pathogens and outcomes. Expert Rev Anti Infect Ther 2012; 10:701-706.

6 Lee WL, Slutsky AS: Sepsis and endothelial permeability. N Engl J Med 2010;363:689691.

7 Aird WC: The role of the endothelium in severe sepsis and multiple organ dysfunction syndrome. Blood 2003;101:3765-3777.

8 Boos CJ, Goon PK, Lip GY: The endothelium, inflammation, and coagulation in sepsis. Clin Pharmacol Ther 2006;79:20-22.

9 Shapiro NI, Schuetz P, Yano K, Sorasaki M, Parikh SM, Jones AE, et al: The association of endothelial cell signaling, severity of illness, and organ dysfunction in sepsis. Crit Care 2010;14:R182.

10 Khakpour S, Wilhelmsen K, Hellman J: Vascular endothelial cell Toll-like receptor pathways in sepsis. Innate Immun 2015;21:827846.

11 Liu SF, Malik AB: NF-kappa B activation as a pathological mechanism of septic shock and inflammation. Am J Physiol Lung Cell Mol Physiol 2006;290:L622-L645.
12 Pahl HL: Activators and target genes of Rel/ NF-kappaB transcription factors. Oncogene 1999; 18:6853-6866.

13 Moser J, Heeringa P, Jongman RM, Zwiers PJ, Niemarkt AE, Yan $\mathrm{R}$, et al: Intracellular RIG-I signaling regulates TLR4-independent endothelial inflammatory responses to endotoxin. J Immunol 2016;196:4681-4691.

14 Miyamoto M, Fujita T, Kimura Y, Maruyama M, Harada H, Sudo Y, et al: Regulated expression of a gene encoding a nuclear factor, IRF-1, that specifically binds to IFN-beta gene regulatory elements. Cell 1988;54:903-913.

15 Schaper F, Kirchhoff S, Posern G, Koster M, Oumard A, Sharf R, et al: Functional domains of interferon regulatory factor I (IRF-1). Biochem J 1998;335:147-157.

16 Dou L, Liang HF, Geller DA, Chen YF, Chen $\mathrm{XP}$ : The regulation role of interferon regulatory factor-1 gene and clinical relevance. Hum Immunol 2014;75:1110-1114.

17 Schwartz JL, Shajahan AN, Clarke R: The role of interferon regulatory factor-1 (IRF1) in overcoming antiestrogen resistance in the treatment of breast cancer. Int J Breast Cancer 2011;2011:912102.

18 Wang Y, John R, Chen J, Richardson JA, Shelton JM, Bennett M, et al: IRF-1 promotes inflammation early after ischemic acute kidney injury. J Am Soc Nephrol 2009;20:1544-1555.

19 Senaldi G, Shaklee CL, Guo J, Martin L, Boone T, Mak TW, et al: Protection against the mortality associated with disease models mediated by TNF and IFN-gamma in mice lacking IFN regulatory factor-1. J Immunol 1999;163: 6820-6826.

20 Venkatesh D, Ernandez T, Rosetti F, Batal I, Cullere X, Luscinskas FW, et al: Endothelial TNF receptor 2 induces IRF1 transcription factor-dependent interferon-beta autocrine signaling to promote monocyte recruitment. Immunity 2013;38:1025-1037.
21 Wang C, Qin L, Manes TD, Kirkiles-Smith NC, Tellides G, Pober JS: Rapamycin antagonizes TNF induction of VCAM-1 on endothelial cells by inhibiting mTORC2. J Exp Med 2014;211:395-404.

22 Jin E, Liu J, Suehiro J, Yuan L, Okada Y, Nikolova-Krstevski V, et al: Differential roles for ETS, CREB, and EGR binding sites in mediating VEGF receptor 1 expression in vivo. Blood 2009;114:5557-5566.

23 van Meurs M, Kurniati NF, Wulfert FM, Asgeirsdottir SA, de Graaf IA, Satchell SC, et al: Shock-induced stress induces loss of microvascular endothelial Tie2 in the kidney which is not associated with reduced glomerular barrier function. Am J Physiol Renal Physiol 2009;297:F272-F281.

24 Su ZZ, Sarkar D, Emdad L, Barral PM, Fisher PB: Central role of interferon regulatory factor-1 (IRF-1) in controlling retinoic acid inducible gene-I (RIG-I) expression. J Cell Physiol 2007;213:502-510.

25 Kowalski PS, Zwiers PJ, Morselt HW, Kuldo JM, Leus NG, Ruiters $\mathrm{MH}$, et al: AntiVCAM-1 SAINT-O-Somes enable endothelial-specific delivery of siRNA and downregulation of inflammatory genes in activated endothelium in vivo. J Control Release 2014; 176:64-75.

26 Wang F, Xia W, Liu F, Li J, Wang G, Gu J: Interferon regulator factor $1 /$ retinoic inducible gene I (IRF1/RIG-I) axis mediates 25-hydroxycholesterol-induced interleukin-8 production in atherosclerosis. Cardiovasc Res 2012;93:190-199.

27 Liu F, Wu S, Ren H, Gu J: Klotho suppresses RIG-I-mediated senescence-associated inflammation. Nat Cell Biol 2011;13:254-262.

28 Collins T, Read MA, Neish AS, Whitley MZ, Thanos D, Maniatis T: Transcriptional regulation of endothelial cell adhesion molecules: NFKB and cytokine-inducible enhancers. FASEB J 1995;9:899-909. 
29 Neish AS, Read MA, Thanos D, Pine R, Maniatis T, Collins T: Endothelial interferon regulatory factor 1 cooperates with NF-kappa B as a transcriptional activator of vascular cell adhesion molecule 1. Mol Cell Biol 1995; 15: 2558-2569.

30 Dagia NM, Harii N, Meli AE, Sun X, Lewis CJ, Kohn LD, Goetz DJ: Phenyl methimazole inhibits TNF- $\alpha$-induced VCAM- 1 expression in an IFN regulatory factor-1-dependent manner and reduces monocytic cell adhesion to endothelial cells. J Immunol 2004;173:2041-2049.
31 DeVerse JS, Sandhu AS, Mendoza N, Edwards CM, Sun C, Simon SI, Passerini AG: Shear stress modulates VCAM-1 expression in response to TNF- $\alpha$ and dietary lipids via interferon regulatory factor- 1 in cultured endothelium. Am J Physiol Heart Circ Physiol 2013; 305:H1149-H1157.

32 Warfel JM, Agnillo FD: Anthrax lethal toxin enhances TNF-induced endothelial VCAM-1 expression via an IFN regulatory factor-1-dependent mechanism: J Immunol 2008; 180: 7516-7524.
33 Dvorin EL, Jacobson J, Roth SJ, Bischoff J: Human pulmonary valve endothelial cells express functional adhesion molecules for leukocytes. J Heart Valve Dis 2003;12:617-624.

34 Dixit E, Bouland S, Zhang Y, Lee AS, Shum B, Hacohen N, et al: Peroxisomes are signaling platforms for antiviral innate immunity. Cell 2010;141:668-681.

35 Marshall JC: Why have clinical trials in sepsis failed? Trends Mol Med 2014;20:195-203. 